\title{
Doping dependence of electronic structure of infinite-layer $\mathrm{NdNiO}_{2}$
}

\author{
Zhao Liu, ${ }^{1,2}$ Chenchao $\mathrm{Xu}^{2,},{ }^{2}$ Chao Cao,,${ }^{3}$, W. Zhu, ${ }^{2}$ Z. F. Wang, ${ }^{1}$ and Jinlong Yang ${ }^{1,0}$ \\ ${ }^{1}$ Hefei National Laboratory for Physical Sciences at the Microscale, \\ University of Science and Technology of China, Hefei, Anhui 230026, China \\ ${ }^{2}$ Westlake Institution of Advanced Study, Westlake University, Hangzhou 300024, China \\ ${ }^{3}$ Hangzhou Key Laboratory of Quantum Matter, \\ Hangzhou Normal University, Hangzhou 310036, China
}

\begin{abstract}
We investigate the electronic structure of nickelate superconductor $\mathrm{NdNiO}_{2}$ upon hole doping, by means of density-functional theory and dynamical mean-field theory. We demonstrate the strong intrinsic hybridization between strongly correlated states formed by Ni-3 $d_{x^{2}-y^{2}}$ orbital and itinerant electrons due to Nd-5d and Ni-3 $d_{z^{2}}$ orbitals, producing a valence-fluctuating correlated metal as the normal state of hole-doped $\mathrm{NdNiO}_{2}$. The Hund's rule appears to play a dominating role on multi-orbital physics in the lightly doped compound, while its effect is gradually reduced by increasing the doping level. Crucially, the hole-doping leads to intricate effects on Ni-3d orbitals, such as a non-monotonic change of electron occupation in lightly doped level, and a flipping orbital configuration in the overdoped regime. Additionally, we also map out the topology of Fermi surface at different doping levels. These findings render a preferred window to peek into electron pairing and superconductivity.
\end{abstract}

Introduction.-Recent discovery of superconductivity in hole-doped infinite-layer $\mathrm{NdNiO}_{2}$ adds the nickelate material to the family of unconventional superconductivity [1]. While the crystal structure of $\mathrm{NdNiO}_{2}$ is similar to the infinite-layer copper oxide superconductors, the electronic structure and magnetic properties of $\mathrm{NdNiO}_{2}$ are very different, which triggers intense on-going discussion. Especially, the $\mathrm{O}-2 p$ states are deeply below the Fermi-level, which points to a Mott insulator for $\mathrm{NdNiO}_{2}[2$ [5]. The strong insulating behavior is suppressed by the self-doping effect due to hybridization between $\mathrm{Nd}-5 d$ conduction electrons and $\mathrm{Ni}-3 d$ local orbitals [6, 7], producing a bad metal observed in transport measurements. Additionally, the related magnetic properties can be understood within the similar route $[8$ - 12]. Besides these studies on the parent compound, the microscopic origin for superconductivity is still elusive [13 19]. Since the nature of normal state may be responsible for electron pairing and superconductivity, it is important to determine their charge doping evolution and connection with superconductivity 20 22].

Let us recall the effect of hole doping on the wellknown copper oxide superconductors where $\mathrm{Cu}-3 d$ local orbitals is mediated by the $\mathrm{O}-2 p$ orbitals. A novel consequence is, upon holes doping most of holes reside on the $\mathrm{O}-2 p$ orbitals [23]. The holes in $\mathrm{O}-2 p$ orbitals will largely weaken the anti-ferromagnetic ordering and induce strong magnetic fluctuations, driving the system into $d$-wave superconductivity [24, 25]. Moreover, since the spins on $\mathrm{O}-2 p$ orbitals strongly interact with local spin on $\mathrm{Cu}-3 d$ states and form the $\mathrm{Cu}-\mathrm{O}$ spin singlet (Zhang-Rice state) [26], a singleband $t-J$ model is believed to capture essential physics in cuprates [27]. Since the dynamics of holes play an important role in possible mechanism for superconductivity in cuprates, it immediately rises questions: Does the hole-doping play a similar role in $\mathrm{NdNiO}_{2}$ ? If not, what is the nature of normal state of hole-doped $\mathrm{NdNiO}_{2}$ ?
To address these questions, one needs the electron occupation and spin information on multi-orbitals using unbiased computations. Despite extensive investigations [28 37], many open issues still remains, waiting for critical justification. For example, the hole doping is usually simulated by the shifts of the energy bands [39], nevertheless the possible electronic state induced by doping $\mathrm{Sr}$ has not been well addressed. Moreover, to avoid the discussion of $f$-electrons on Neodymium [38], most of existing literatures made a detour and studied $\mathrm{LaNiO}_{2}$ alternatively, but these calculations are at odds with the experimental fact that no superconductivity is found in $\mathrm{LaNiO}_{2}[1]$. In a word, a full-electron ab initio simulation of hole-doped $\mathrm{NdNiO}_{2}$ is still outstanding.

In this work, we aim at the normal state of $\mathrm{NdNiO}_{2}$ by performing both density functional theory (DFT) and dynamical mean field theory (DMFT) calculations. Firstly, we build a realistic doping model to study the effect of Sr doping continuously and find that the evolution of the electronic states upon hole doping is non-rigid-band like: the electron pocket at $\Gamma$ point vanishes quickly, and the Fermi level is almost pinned by the Ni-3 $3 d_{x^{2}-y^{2}}$ orbital. Then we perform a many-body calculation on the normal state of holedoped $\mathrm{NdNiO}_{2}$ without considering the $f$-orbitals, and compare with the results from full electron many-body calculations by explicitly considering Nd- $4 f$ electrons. We identify several key features in the evolution of electronic structure upon hole doping: 1) While $d^{9}$ state is the most probable, the weight of $d^{8}$ is much larger than $d^{10} \underline{\mathrm{L}}$ ( $\underline{\mathrm{L}}$ means a hole on ligand), which points to a multi-orbital physics rather than the charge-transfer picture; 2) The total occupation of Ni-3d state is close to 8.6, thus lightly doped compound is a valencefluctuating correlated metal; 3) The Hund's rule plays an important role in the lightly doped compound, and its effect is gradually reduced upon hole doping; 4) There is a non-monotonic doping effect in Ni-3d orbital configuration; 5) Significant band renormaliza- 
(a)

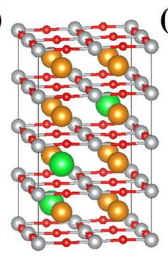

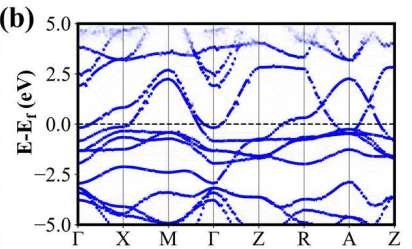

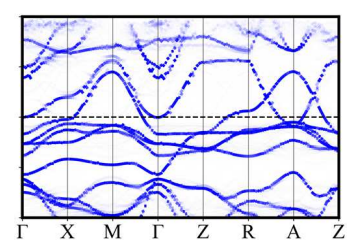

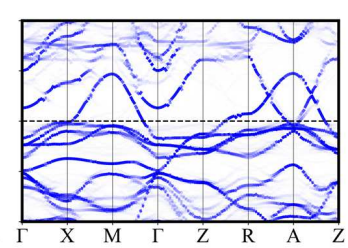

Figure 1. (a) Perspective view of $\mathrm{NdNiO}_{2}$ supercell with $\delta=18.75 \%$. The silver, red, yellow and green balls represent $\mathrm{Ni}, \mathrm{O}, \mathrm{Nd}$ and $\mathrm{Sr}$ atoms, respectively. (b) Unfolded band structures for different $\mathrm{Sr}$ doping concentration. From left to right: $\delta=0.00 \%, 6.25 \%, 18.75 \%$ and $31.25 \%$.

tion is observed for the Ni-3d $d_{x^{2}-y^{2}}$ state, with mass enhancement of $\sim 2.4$. It is quickly suppressed to $\sim$ 2.1 upon heavy hole-doping. We believe these findings are helpful to understand the unconventional superconductivity in this system. For instance, the correlated Ni-3 $d_{x^{2}-y^{2}}$ orbital is most relevant for electron pairing, and the change of topology of Fermi surface could result in disappearance of superconductivity.

Methods.-DFT calculation was performed with the plane wave projector augmented wave method implemented in the Vienna $a b$ initio simulations package (VASP) 40 42]. The Perdew-Burke-Ernzerhof version of generalized gradient approximation (PBE) was applied [43]. To simulate the effect of Sr doping, 1, 3 and $5 \mathrm{Nd}$ atoms are replaced by $\mathrm{Sr}$ atoms in a $2 \times 2 \times 4$ supercell containing $16 \mathrm{Nd}, 16 \mathrm{Ni}$ and $32 \mathrm{O}$ atoms, as depicted in Fig. 1(a). In this way, we can consistently mimic the hole doping concentration $(\delta)$ at $6.25 \%$, $18.75 \%$ and $31.25 \%$ lying at the underdoped, optimal doping and overdoped regions [20, 21]. Considering the fact that the thin films are grown on $\mathrm{SrTiO}_{3}(001)$ surface, the lattice constants in the ab plane of supercell cell are fixed to $a=b=3.92 \AA$ while the lattice constant in the $c$ direction and atomic positions are allowed to relax. For band structure calculation, we choose the following high symmetric path $\Gamma(0,0,0)$ $\mathrm{X}(\pi, 0,0)-\mathrm{M}(\pi, \pi, 0)-\Gamma-\mathrm{Z}(0,0, \pi)-\mathrm{R}(\pi, 0, \pi)-\mathrm{A}(\pi, \pi$, $\pi)-\mathrm{Z}$ (see Fig. 1(a)). To show the effect of Sr doping on electronic structures, all the band structures are unfolded to the unit cell by PyProcar package [44].

DFT + DMFT calculations were performed using the code EDMFTF, developed by Haule et al. 45] based on the Wien2k package [46]. Similar to prior studies, we replace $\mathrm{Nd}$ with La in our calculations, and the doping effect is simulated by removing conduction electrons in the spirit of virtual crystal approximation (VCA) 48]. Charge self-consistency is enforced throughout the calculations. The on-site Coulomb interaction parameters for Ni-3d orbitals are chosen to be $F^{0}=6.0 \mathrm{eV}, F^{2}=7.754 \mathrm{eV}$, and $F^{4}=4.846 \mathrm{eV}$, respectively, or equivalently $U=6.0 \mathrm{eV}$ and $J=0.9 \mathrm{eV}$. The local impurity problem is solved using continuoustime quantum Monte Carlo (CTQMC) method [49] at $\beta=100 \mathrm{eV}^{-1}$ or $116 \mathrm{~K}$. For each impurity problem, we used $4 \times 10^{9}$ total CTQMC steps in each DMFT iteration, and self energies from last 5 iterations after convergence were averaged and analytically continued to obtain real-frequency self energy $\Sigma(\omega)$ using maximum entropy method.

DFT results. - We first calculate the lattice constant in the $c$ direction (see Fig. S1), and observe a linear relation between $\mathrm{Sr}$ doping concentration and $c$, in accordance with experimental result [20]. After structural optimization, $\mathrm{NiO}_{2}$ plane is no longer exactly flat as in the pristine compound. To measure the roughness of the plane, we define roughness as $R=\max \left\{\delta_{c}\right\}$, where $\delta_{c}$ is the buckling of each $\mathrm{NiO}_{2}$ plane in the supercell (in unit of $\AA$ ). As displayed in Fig. S1, $R$ is less than $0.03 \AA$ even at large $\delta=31.25 \%$, therefore the effect of structure distortion on electronic structure is secondary, making possible to prepare atomically flat surface for scanning tunneling microscope [22].

The unfolded band structures at different $\delta$ are shown in Fig. 1(b), where the band structure of pristine compound is also plotted to guide the eye. To facilitate our discussion, we label the Fermi pockets as $\alpha, \beta$ and $\gamma$ for the dominating sheet, pocket at $\Gamma$ point and pocket at A point in the pristine compound [8, 22]. These pockets behave differently to $\mathrm{Sr}$ doping. Among these Fermi pockets, $\beta$ pocket is very sensitive to $\mathrm{Sr}$ doping and almost vanishing at a low doping level $\delta=$ $6.25 \%$. Since it is contributed by $\mathrm{Nd} d_{z^{2}}$ and $\mathrm{Ni} d_{z^{2}}$ orbitals, their density of states (DOS) gradually decrease to zero as shown in Fig. 2(a) and 2(d). When $\delta$ increases to $31.25 \%$, the $\beta$ pocket has experienced a blue-shift up to $0.86 \mathrm{eV}$. Such a large shift indicates the free-particle nature of this pocket. The $\gamma$ pocket, which has smaller effective electron mass and thus more itinerant, nevertheless has smaller blue-shift than $\beta$ pocket and does not vanish even at $\delta=31.25 \%$. In a freeparticle picture, a homogeneous holes doping will lead to the drop of Fermi level, namely, all the bands are rigid and have the same blue-shift. The smaller blueshift of the more itinerant $\gamma$ pocket reflects that such a pocket is actually more correlated. As we will show below, such a correlation originates from the Hund's coupling between Ni-3d for this pocket is mainly formed by the $\mathrm{Ni} d_{x z}$ and $d_{y z}$ orbitals [8]. As for the $\alpha$ sheet, its blue-shift strongly depends on $\delta$. At smaller $\delta$, it does not shift until the vanishing of $\beta$ pocket(see Fig. 2(c)). After that, it slowly undergoes blue-shift together with $\gamma$ pocket. Moreover, since $\alpha$ sheet wraps around a large volume, its blue-shift is quite small. In other words, the Fermi level is pinned to $\alpha$ sheet. Con- 

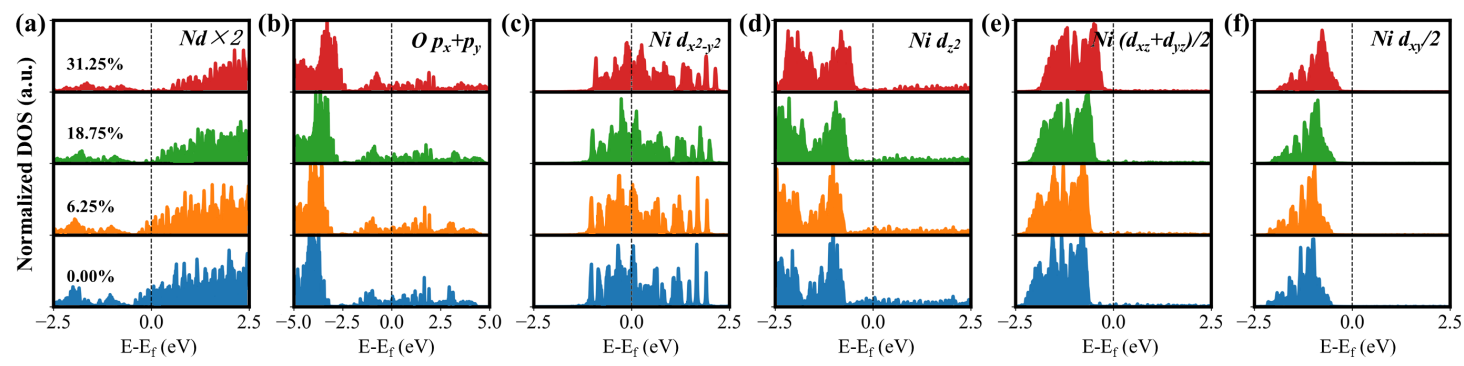

Figure 2. (a)-(d) Projected density of state with different $\delta$ on different subspaces, from left to right: Nd atoms, O $p_{x+y}$ orbitals, Ni $d_{x^{2}-y^{2}}$ orbitals, Ni $d_{z^{2}}$ orbitals, Ni $d_{x z}+d_{y z}$ orbitals and Ni $d_{x y}$ orbitals. The weight in (a), (e) and (f) has been rescaled by a factor of $2,1 / 2$ and $1 / 2$. From bottom to top, $\delta$ increases from $0.00 \%$ to $31.25 \%$ in each figure.

sider the fact that $\alpha$ sheet are mainly contributed by Ni $d_{x^{2}-y^{2}}$ orbital, such a Fermi surface pinning by Ni $d_{x^{2}-y^{2}}$ orbital leads to two results: 1 ) the charge transfer energy between $\mathrm{Ni} d_{x^{2}-y^{2}}$ and $\mathrm{O}-2 p$ is reduced since O-2 $p$ experiences a large blue-shift (as shown in Fig. 2(b) [50]. Such a decrease in charge transfer energy in $\mathrm{NiO}_{2}$ plane indicates more contributions from $\mathrm{O}-2 p$ orbitals in the Fermi level, which may explain the existence of "shoulder" structure in the O-K edge resolved by electron energy loss spectroscopy (EELS) recently [51]. 2) the splitting between $\mathrm{Ni} d_{x^{2}-y^{2}}$ and other $\mathrm{Ni}$ $d$ orbitals becomes smaller, which drives $\mathrm{NdNiO}_{2}$ towards multi-orbital Hund's physics [52].

We note that the above features upon doping are quite robust since similar behavior has been repeated with SCAN functional (see SM). In addition, vanishingly small DOS of $\mathrm{Sr}$ is identified around the Fermi level, therefore $\mathrm{Sr}$ doping does not introduce any $\mathrm{Sr}$ orbitals near the Fermi level. Thus it is valid to ignore the chemical differences by removing valence electrons while simulating the doping effect.

DFT + DMFT results. - We now turn to manybody results from DFT + DMFT calculations. In Fig. 3. we present the $\mathbf{k}$-resolved spectral function for pristine, 0.2 hole doped, and 0.4 hole doped $\mathrm{NdNiO}_{2}$ from DFT + DMFT calculations. Despite of the relatively high temperature $(116 \mathrm{~K})$, the electron states in the pristine compound are already very coherent near the Fermi level, in accordance with previous reports [9, 47]. The spectral function of the pristine compound is quite similar to the DFT band structure, giving rise to three sheets of Fermi surfaces with similar shapes. Holedoping quickly suppresses the $\beta$ pocket, which disappears with less than 0.1 hole doping. Therefore, this $\mathrm{Nd}-d_{z^{2}} / \mathrm{Ni}-d_{z^{2}}$ derived pocket may be irrelevant to the superconductivity. The $\gamma$ pocket is also substantially reduced by hole-doping, and eventually disappears around 0.3 hole-doping. The largest $\alpha$ pocket is only moderately affected before 0.3 hole-doping, due to its large volume and partial flat-band close to the Fermi level at R. Further hole doping also causes substantial blue-shift of $\alpha$ pocket. We show the calculated DMFT Fermi surfaces in Fig. [S4. In the pristine compound, the occupation of $\alpha, \beta$, and $\gamma$ pockets are
0.98 holes, 0.03 electrons, and 0.02 electrons, respectively. Upon 0.2 hole doping, the occupation of $\alpha$ and $\gamma$ pocket are 1.19 holes and $<0.01$ electrons, respectively, while the $\beta$ pocket disappears. In the 0.4 hole doped compound, only $\alpha$ pocket remains with 1.32 hole occupation. Such observation is also consistent with the above DFT analysis, and follows Luttinger theorem. Moreover, as a result of the large blue-shift of $\beta$ and $\gamma$ pockets upon hole doping, as well as the pinning of the $\alpha$-pocket due to $\mathrm{Ni}-3 d_{x^{2}-y^{2}}$, the hybridization between the itinerant electrons and local $\mathrm{Ni}-3 d$ orbitals reduces, resulting in sharper quasi-particle behavior upon hole-doping [comparing Fig. [3(b,c) with (a) (see also Fig. [55)].

We then focus on the $3 d$ orbital occupations of $\mathrm{Ni}$ atom (Fig. 4(a), also TAB. S-1). The total Ni-3d occupation in the pristine compound is $8.675 e$, thus the $\mathrm{Ni}$ atom is valence fluctuating between $\mathrm{Ni}^{2+}\left(d^{8}\right)$ and $\mathrm{Ni}^{+}$ $\left(d^{9}\right)$. Despite of the consistent and significant changes in the spectral function due to hole doping, the doping effect to orbital occupations clearly exhibits different behavior for small and large doping range. Surprisingly, for small hole doping $(<0.1$ hole/cell), the overall Ni-3d occupation even increases. Once the holedoping exceeds 0.1 hole/cell, the overall Ni-3d occupations follow a consistent linear decreasing behavior with respect to the hole-doping level. Closer examination reveals further details. Among the $5 \mathrm{Ni}-3 d$ orbitals, the $t_{2 g}$ orbitals are nearly fully occupied, and are less relevant to the electronic states near the Fermi level. Therefore, the $t_{2 g}$ orbitals are "frozen" during hole doping, although they cannot be ignored in the discussion of total $d$-occupation. In the following discussion, we focus on the $e_{g}$ orbitals 12,29, 33, 37]. The doped hole will populate on $d_{x^{2}-y^{2}}$, as the occupation decreases with hole doping as shown in Fig. 4(a). Interestingly, $d_{z^{2}}$ occupation increases with hole doping. These findings are at odds with Ref. [32], where the hole mainly goes to $d_{z^{2}}$ orbital. Such a charge correlation between $d_{z^{2}}$ and $d_{x^{2}-y^{2}}$ agrees with the band shift upon hole doping from DFT calculations. In addition, the hole doping has nontrivial effect on the effective mass of the Ni-3d orbitals as well. As shown in Tab. S-1. Ni- $3 d_{x^{2}-y^{2}}$ orbital has the largest $m^{*} / m \sim 2.4$, 


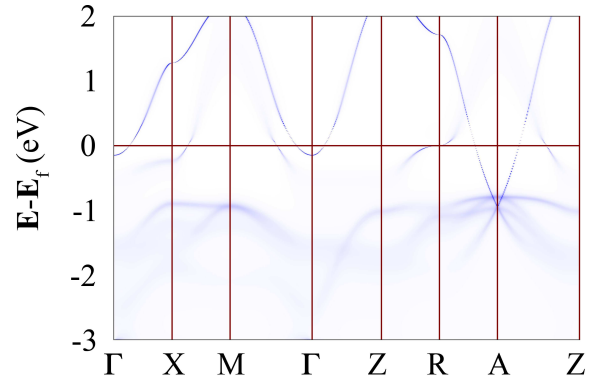

(a)

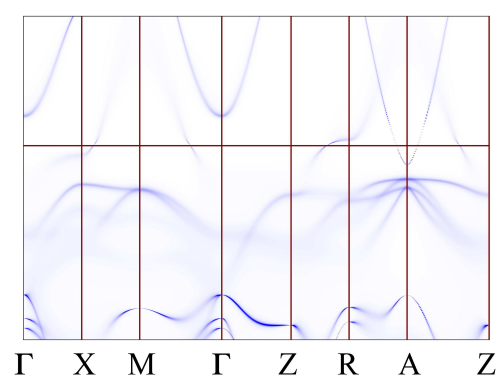

(b)

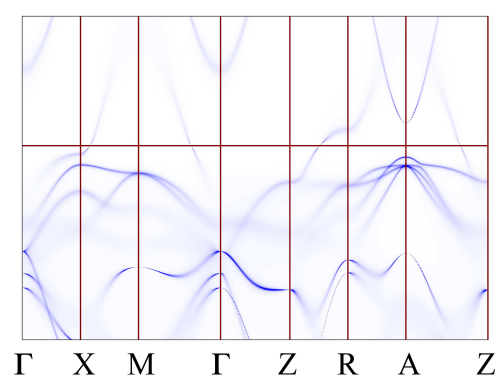

(c)

Figure 3. k-resolved spectral function from DFT+DMFT calculations for (a) pristine, (b) 0.2 hole-doped, and (c) 0.4 hole-doped $\mathrm{NdNiO}_{2}$ at $116 \mathrm{~K}$.

and all other $d$ orbitals show only mild correlation effect with $m^{*} / m$ around 1.3 in the pristine compound. While the later is less affected by hole doping, the effective mass of $d_{x^{2}-y^{2}}$, which is believed to be most relevant to the superconductivity, strongly depends on the doping. It ranges between 2.4 to 2.6 within 0.3 hole/cell doping, and suddenly drops to $\sim 2.1$ with 0.4 hole/cell doping.

We also plot the statistical weight of sampled manybody states in Fig. 4(b). Again, we see clearly distinct hole-doping behavior for the small and large doping range. For the small doping range $(<0.1$ hole/cell $)$, the $d^{9}$ weight is significantly enhanced, primarily due to large increase of $d_{x^{2}-y^{2}}^{9}$ weight. Noticing that $d_{x^{2}-y^{2}}^{9}$ denotes $d^{9}$ with unpaired $d_{x^{2}-y^{2}}$, this is consistent with the reduced $d_{x^{2}-y^{2}}$ occupation due to hole doping. Beyond 0.1 hole/cell doping, the $d^{9}$ weight consistently drops, whereas the $d_{x^{2}-y^{2}}^{9}$ weight gradually reaches saturation around $43 \%$ at 0.4 hole/cell. Similarly, the $d^{8}$ configuration weight reduces from $32.7 \%$ to $32.1 \%$ in the small doping range, then linearly increases to $33.9 \%$ at 0.4 hole/cell doping. Nevertheless, within the doping range we investigated, the weight of $d^{8}$ configuration is always more than 3-times larger than $d^{10} \underline{\underline{L}}$, and even enhances with 0.4 holedoping. In a typical charge-transfer insulator, it is the weights of $d^{10} \underline{\mathrm{L}}$ that should be comparable to that of $d^{9}$ rather than $d^{8}[29]$. Thus it suggests the multi-orbital nature rather than charge-transfer. Moreover, in the pristine and small doping cases, the spin-triplet states always dominate the $d^{8}$ states (almost twice the weight of spin-singlet states). However, the weight ratio between spin-triplet and singlet states quickly reduces, and becomes close to 1 in the 0.4 hole/cell doped case. Therefore, the Hund's physics is important in the pristine and lightly-doped system, but may lose its importance in the heavily doped compounds.

Such non-monotonic doping effect is also observed for the crystal-field splitting of the Ni-3d electrons. Bare atomic level of Ni-3d orbitals (with respect to the Fermi level) can be obtained by fitting the undoped DFT result to a tight-binding Wannier Hamiltonian, which yields $-1.398 \mathrm{eV},-1.459 \mathrm{eV},-1.676 \mathrm{eV}$, and -1.919 $\mathrm{eV}$ for $d_{x^{2}-y^{2}}, d_{z^{2}}, d_{z x / z y}, d_{x y}$ orbitals, respectively. The $e_{g}$ orbitals are nearly degenerate and is separated from $t_{2 g}$ by a large gap. Such crystal field splitting is consistent with the fact that the $t_{2 g}$ are "frozen" during hole doping. In the DFT + DMFT calculations, the electron density and energies are renormalized, and the orbitals order as $E\left(d_{z^{2}}\right)>E\left(d_{x^{2}-y^{2}}\right)>E\left(d_{z x / z y}\right)$ $>E\left(d_{x y}\right)$ in the pristine compound. Since $d_{z^{2}}$ has larger occupation than $d_{x^{2}-y^{2}}$, the orbital order flip between $d_{z^{2}}$ and $d_{x^{2}-y^{2}}$ reflects the fact that $d_{x^{2}-y^{2}}$ has much larger onsite Hubbard repulsion than $d_{z^{2}}$, which renders the validity of the one-band Hubbard model 37. Since $d_{x^{2}-y^{2}}$ occupation is larger than halffilled, the residue of hole on $d_{x^{2}-y^{2}}$ will greatly stabilize the system as found in Fig. 4 a. The observation of stronger correlation of $d_{x^{2}-y^{2}}$ than $d_{z^{2}}$ is also in agreement with the fact that $d_{x^{2}-y^{2}}$ has a much larger effect mass than $d_{z^{2}}$. Normally, for $\mathrm{D}_{4 h}$ symmetry without apical anions, crystal field splitting leads to lowest $d_{z x / z y}, d_{z^{2}}$ instead of $d_{x y}$ orbitals. However, the $\mathrm{NdNiO}_{2}$ compound stems from $\mathrm{NdNiO}_{3}$ crystal, where the octahedral crystal-field leads to degenerate $t_{2 g}$ and $e_{g}$ splittings for $\mathrm{Ni}-3 d$ orbitals. Once the apical $\mathrm{O}^{2-}$ anions are removed, negative charge occupation accumulates at the apical oxygen position [4, 34], and thus orbitals extending along the $z$-direction costs energy, raising energies of $d_{z^{2}}, d_{z x}$ and $d_{z y}$ orbitals. Therefore, the $d_{x y}$-orbital becomes the lowest-lying component. The $d_{z x / z y}$ orbitals are less affected than the $d_{z^{2}}$, and therefore are also low-lying and nearly fully occupied. In such distorted octahedral crystal field, the original octahedral crystal-field splitting is characterized by the energy difference between $d_{x^{2}-y^{2}}$ and $d_{x y}$ orbitals $\Delta_{0}=E\left(d_{x^{2}-y^{2}}\right)-E\left(d_{x y}\right)$, and the effect of distortion is reflected in the energy difference between $d_{x y}$ and $d_{z x / z y}$ orbitals $\Delta_{1}=E\left(d_{z x / z y}\right)-E\left(d_{x y}\right)$. We plot the doping dependent $\Delta_{0}$ and $\Delta_{1}$ in Fig. 4(c). Throughout the hole doping range, $\Delta_{0}$ exhibits linear dependence on the doping level, but $\Delta_{1}$ decreases below 0.1 hole doping, and then quickly linearly increases with further hole-doping. This leads to an inversion between $d_{x^{2}-y^{2}}$ and $d_{z x / z y}$ at 0.4 hole doping.

Finally, we address the effect of undetermined double counting term. The above reported results were obtained using a fixed double-counting $V_{d c}=47.4 \mathrm{eV}$ (or equivalently nominal $n^{0}=9.0$ ), which leads to actual $n^{d}$ close to 8.6. A $n^{d}=9.0$ solution can be 

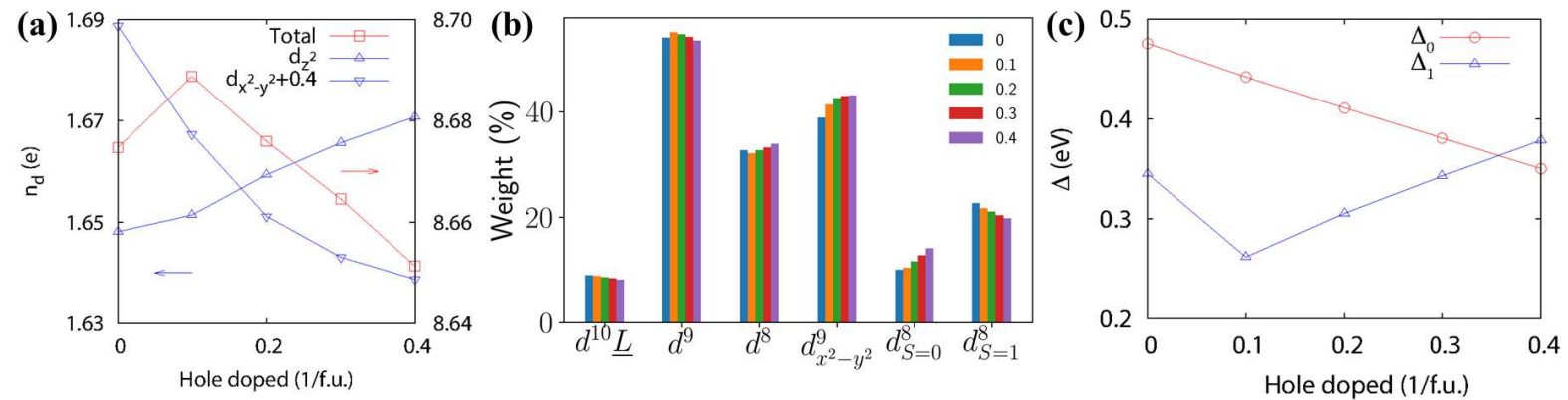

Figure 4. Evolution of (a) Ni-3d $e_{g}$ orbital occupation, (b) weight of many-body states, and (c) Ni-3d orbital crystal field splitting under hole doping. In panel (a), the occupation of $d_{x^{2}-y^{2}}$ is increased by 0.4 to fit in the plot. In panel (b), $d_{x^{2}-y^{2}}^{9}$ indicates $d^{9}$ state with unpaired $d_{x^{2}-y^{2}}$ electron, $d_{S=0}^{8} / d_{S=1}^{8}$ are $d^{8}$ spin-singlet/spin-triplet states, respectively. In panel (c), $\Delta_{0}\left(\Delta_{1}\right)$ are the energy differences between $d_{x^{2}-y^{2}}\left(d_{z x / z y}\right)$ and $d_{x y}$ orbitals, respectively.

achieved by using $V_{d c}=51.84 \mathrm{eV}$. In this case, the $\beta$ pocket disappears even in the pristine compound, and the size of $\gamma$ pocket is drastically reduced compared to the above results. Hole-doping in this case will quickly suppress the $\gamma$ pocket, leaving only $\alpha$ pocket in the BZ. The $d^{10} \underline{\mathrm{L}}$ configuration weight is much enhanced and can be comparable to the $d^{8}$ weight (Tab. S-III). In this case, the hole doping quickly suppress the effect of Hund's rule, and the weight of spin-singlet states are comparable to the spin-triplet states as early as 0.2 hole/cell doping. Nevertheless, the aforementioned non-monotonic doping effect to the $d$-orbital occupation, many-body configuration weight, crystalfield splitting and effective mass still remains (Fig. S6. Tab. S-II] Tab. S-III). Thus, our results are robust with respect to the choice of double counting terms.

Summary and Discussion. - We have performed a systematic study of electronic properties of the normal state of $\mathrm{NdNiO}_{2}$ by DFT and DMFT methods. The complementary methods show the normal state of hole-doped $\mathrm{NdNiO}_{2}$ as a valence-fluctuating correlated metal. The Hund's effect and hybridization effect gradually reduces by increasing the doping level. Importantly, we identify a non-monotonic many-body doping effect on Ni-3d orbitals, as evidenced by the electron occupation, orbital crystal field splitting, and statistical spectral weight of orbital configurations. Additionally, the doping effect leads to the change of topology of Fermi surface and reduction of correlations, which could be relevant to the underlying superconductivity 22.

We would like make several remarks here. First of all, our results show the normal state of doped $\mathrm{NdNiO}_{2}$ is quite different from the copper oxide superconductors, instead it is closer to the iron-based superconductors where multi-orbital physics dominates. Second, we identify Ni-3 $3 d_{x^{2}-y^{2}}$ orbital as the most correlated one, which should be helpful for building the effective model. Third, some features shown here could provide plausible understanding for experimental observations. For example, both DFT and DFT + DMFT calculations (Fig. 2]and 3) show $\mathrm{O}-2 p$ state moving towards Fermi level upon Sr doping, which is consistent with a recent
EELS study on O-K edge that gives a "shoulder" structure pointing to Ni-O singlet state 51. Finally, we argue that the $\mathrm{Nd} f$-electrons influence the electronic states below the Fermi energy, leaving the physics close to the Fermi level unaffected. In addition, the occupations and effective masses of Ni-3d orbitals in both pristine and hole-doped compounds are qualitatively similar to the case without $f$-electrons. These arguments are supported by our full-electron calculation of $\mathrm{NdNiO}_{2}$ by explicitly including $\mathrm{Nd}-4 f$ orbitals (see Supplemental materials Tab. [S-IV] FIG. [S8). These results all indicates the $f$-electrons may be irrelevant in $\mathrm{NdNiO}_{2}$, in good agreement with the existing experiment [53].

Acknowledgement. - The authors thanks J.-X. Zhu, Zhi Ren, and H. H. Wen for inspiring and helpful discussions. This work was supported by NSFC (No. 11774325, 11874137, 21603210, 21603205, 21688102), National Key Research and Development Program of China (No. 2017YFA0204904, 2016YFA0200604), Anhui Initiative in Quantum Information Technologies (No. AHY090400), Fundamental Research Funds for the Central Universities and the Start-up Funding from Westlake University. We thank Supercomputing Center at USTC for providing the computing resources. DMFT calculations were performed on the High Performance Computing Cluster at Hangzhou Normal University, as well as at the Beijing Supercloud Computing Center.

Note added. - In the final stage of this work, we become aware of recent work [63] using GW + DMFT and [55, 56] using DFT +DMFT.

* E-mail: ccao@hznu.edu.cn

† E-mail: jlyang@ustc.edu.cn

[1] D. Li, K. Lee, B. Y. Wang, M. Osada, S. Crossley, H. R. Lee, Y. Cui, Y. Hikita, H. Y. Hwang, Nature 572, 624-627 (2019).

[2] A. S. Botana, M. R. Norman, Phys. Rev. X 10, 011024 (2020).

[3] H. Sakakibara, H. Usui, K. Suzuki, T. Kotani, H. Aoki 
and K. Kuroki, Phys. Rev. Lett. 125, 077003 (2020).

[4] Y. Nomura, M. Hirayama, T. Tadano, Y. Yoshimoto, K. Nakamura and R. Arita, Phys. Rev. B 100, 205138 (2019).

[5] P. Jiang, L. Si, Z. Liao and Z. Zhong, Phys. Rev. B 100, 201106(R) (2020).

[6] M. Hepting et al. Nat. Mater. 19, 381-385 (2020).

[7] G.-M. Zhang, Y.-F. Yang and F.-C. Zhang, Phys. Rev. B 101, 020501 (2020).

[8] Z. Liu, Z. Ren, W. Zhu, Z. F. Wang and J. Yang, npj Quantum Mater. 5, 31 (2020).

[9] L. Leonov, S. L. Skornyakov and S. Y. Savrasov, Phys. Rev. B 101, 241108(R) (2020).

[10] M.-Y. Choi, W. E. Pickett and K.-W. Lee, arXiv: 2005.03234

[11] I. Leonov and S. Y. Savrasov, arXiv: 2006.05295

[12] X. Wan, V. Ivanov, G. Resta, I. Leonov and S. Y. Savrasov, arXiv: 2008.07465

[13] M. Jiang, M. Berciu and G. A. Sawatzky Phys. Rev. Lett. 124, 207004 (2020).

[14] X. Wu, D. D. Sante, T. Schwemmer, W. Hanke, H. Y. Hwang, S. Raghu and R. Thomale, Phys. Rev. B 101, 060504(R) (2020).

[15] Y.-H. Zhang and A. Vishwanath, Phys. Rev. Res. 2, $023112(2020)$.

[16] P. Werner and S. Hoshino, Phys. Rev. B 101, 041104(R) (2020).

[17] L.-H. Hu and C. Wu, Phys. Rev. Res. 1, 032046(R) (2019).

[18] J. Chang, J. Zhao and Y. Ding, arXiv: 1911.12731

[19] P. Adhikary, S. Bandyopahyay, T. Das, I. Dasgupta and T. Saha-Dasgupta, arXiv: 2005.01243

[20] D. Li, B. Y. Wang, K. Lee, S. P. Harvey, M. Osada, B. H. Goodge, L. F. Kourkoutis and H. Y. Hwang, Phys. Rev. Lett. 125, 027001 (2020).

[21] S. Zeng et al., arXiv: 2004.11281.

[22] Q. Gu et al, arXiv: 2006.13123

[23] H. Romberg, M. Alexander, N. Nücker, P. Adelmann and J. Fink, Phys. Rev. B 42, 8768-8771 (1990).

[24] P. W. Anderson, Science 235, 1196 (1987).

[25] P. A. Lee, N. Nagaosa and X.-G. Wen, Rev. Mod. Phys. 78, 17-85 (2006).

[26] F. C. Zhang and T. M. Rice, Phys. Rev. B 37, 37593761 (1988).

[27] M. Ogata and H. Fukuyama, Rep. Prog. Phys. 71, 036501 (2008).

[28] Z.-J. Lang, R. Jiang and W. Ku, arXiv: 2005.00022

[29] J. Karp, A. S. Botana, M. R. Norman, H. Park, M. Zingl and A. Millis, Phys. Rev. X 10, 021061 (2020).

[30] H. Zhang, L. Jin, S. Wang, B. Xi, X. Shi, F. Ye and J.-W. Mei, Phys. Rev. Res. 2, 013214 (2020).

[31] V. Olevano, F. Bernardini, X. Blase and A. Cano, Phys. Rev. B 101, 161102(R) (2020).

[32] F. Lechermann, Phys. Rev. B 101, 081110(R) (2020).

[33] F. Lechermann, arXiv: 2005.01166

[34] Y. Gu, S. Zhu, X. Wang, J. Hu and H. Chen, Commun. Phys. 3, 84 (2020).

[35] Z. Wang, G.-M. Zhang, Y.-F. Yang and F.-C. Zhang, arXiv: 2006.15928

[36] R. He, P. Jiang, Y. Song, M. Chen, M. Jin, L. Shui and Z. Zhong, Phys. Rev. B 102, 035118 (2020).

[37] M. Kitatani, L. Si, O. Janson, R. Arita, Z. Zhong and K. Held, arXiv:2002.12230.

[38] M.-Y. Choi, K.-W. Lee and W. E. Pickett, Phys. Rev. B 101, 020503 (2020).

[39] J.-C. Gao, Z.-J. Wang, C. Fang and H.-M. Weng, arXiv: 1909.04657

[40] M. P. Teter, M. C. Payne and D. C. Allan, Phys. Rev.
B 40, 12255 (1989).

[41] G. Kresse and J. Furthmüller, Phys. Rev. B 54, 11169 (1996).

[42] P. E. Blöchl, Phys. Rev. B 50, 17953 (1994).

[43] J. P. Perdew, K. Burke and M. Ernzerhof, Phys. Rev. Lett. 77, 3865-3868 (1996).

[44] U. Herath, P. Tavadze, X. He, E. Bousquet, S. Singh, F. Muñoz and A. H. Romero Comp. Phy. Commun. 251, 107080 (2020).

[45] K. Haule, C.-H. Yee and K. Kim, Phys. Rev. B 81, 195107 (2010).

[46] P. Blahha, K. Schwarz, F. Tran, R. Laskowski, G. K. H. Madsen and L. A. Marks, J. Chem. Phys. 152, 074101 (2020).

[47] S. Ryee, H. Yoon, T. J. Kim, M. Y. Jeong and M. J. Han, Phys. Rev. B 101, 064513 (2020).

[48] L. Ballaiche and D. Vanderbilt, Phys. Rev. B 61, 7877 (2000).

[49] E. Gull, A. J. Millis, A. I. Lichtenstein, A. N. Rubtsov, M. Troyer and P. Werner, Rev. Mod. Phys. 83, 349404 (2011).

[50] J. Kirshna, H. LaBollita, A. O. Fumega, V. Pardo and A. S. Botana, arXiv: 2008.02237

[51] B. H. Goodge, D. Li, M. Osada, B. Y. Wang, K. Lee, G. A. Sawatzky, H. Y. Hwang and L. F. Kourkoutis, arXiv: 2005.02847

[52] A. Georges, L. de' Medici and J. Mravlje, Annu. Rev. Condens. Matter Phys. 4, 137-178 (2013).

[53] M. Osada, B. Y. Wang, B. H. Goodge, K. Lee, H. Yoon, K. Sakuma, D. Li, M. Miura, L. F. Kourkoutis, and H. Y. Hwang, Nano Lett. 20, 5735-5740 (2020).

[54] F. Petocchi, V. Christiansson, F. Nilsson, F. Aryasetiawan and P. Werner, arXiv:2006.00394

[55] Y. Wang, C.-J. Kang, H. Miao and G. Kotliar, arXiv: 2006.15305

[56] B. Kang, C. Melnick, P. Semon, G. Kotliar and S. Choi, arXiv: 2007.14610

[57] D. Li, B. Y. Wang, K. Lee, S. P. Harvey, M. Osada, B. H. Goodge, L. F. Kourkoutis and H. Y. Hwang, Phys. Rev. Lett. 125, 027001 (2020).

[58] J. Sun, A. Ruzsinszky and P. Perdew, Phys. Rev. Lett. 115, 036402 (2015).

[59] J. Sun et al. Nat. Chem. 8, 831-836 (2016).

[60] C. Lane, J. W. Furness, I. G. Buda, Y. Zhang, R. S. Markiewicz, B. Barbiellini, J. Sun and A. Bansil, Phys. Rev. B 98, 125140 (2018).

[61] J. W. Furness, Y. Zhang, C. Lane, I. G. Buda, B. Barbiellini, R. S. Markiewicz, A. Bansil and J. Sun, Commun. Phys. 10, 1038 (2018).

[62] Y. Zhang, C. Lane, J. W. Furness, B. Barbiellini, J. P. Perdew, R. S. Markiewicz, A. Bansil and J. Sun, Proc. Natl. Acad. Sci. U.S.A. 117, 68-72 (2020).

[63] F. Petocchi, V. Christiansson, F. Nilsson, F. Aryasetiawan and P. Werner, arXiv:2006.00394 
In this supplementary materials, we provide additional results to support the discussion in the main text.

\section{Structure optimization}

The doping configuration with $\delta=6.25 \%, 18.75 \%$ and $31.25 \%$ is shown in Fig. S1(a). Since $\mathrm{Sr}^{2+}$ has larger ionic radius than $\mathrm{Nd}^{3+}$, the lattice constant in the c direction will expands when $\mathrm{Sr}$ is doped. The calculated lattice constant of c direction with respect to $\delta$ is shown in Fig. S1(b), where lattice constant monotonically increases with $\delta$, in accordance with expeimental data [57]. Since the distortion of $\mathrm{NiO}_{2}$ plane will hugely influence its correlations, we also measure the flatness of each $\mathrm{NiO}_{2}$ plane in the supercell after structural optimization. As shown in Fig. S1(b), the roughness of $\mathrm{NiO}_{2}$ plane is only $0.03 \AA$ at a high doping concentration $\delta=31.25 \%$. Therefore, a atomic flat surface is in principle possible.

\section{Result of SCAN functional}

The recently developed SCAN functional [58] makes it possible to treat charge, spin and lattice degrees of freedom on equal footing. As a parameter-free functional, SCAN has shown great success in different types of bonding systems [59], even in cuprates 60 62], which is believed to be the typical strongly-correlated system. Therefore, we also use SCAN functional to study the effect of Sr doping.

The unfolded band structure at $\delta=0.00 \%, 6.25 \%$, $18.75 \%$ and $31.25 \%$ is shown in Fig. S2. Similar to the result of $\mathrm{PBE}$, the evolution of electronic structure upon hole doping is non-rigid-like: 1) $\beta$ pocket undergoes Lifshitz transition at a low $\delta$ around $6.25 \%$. 2) $\gamma$ pocket does not vanish even at $\delta=31.25 \%$. 3) the $\alpha$ sheet pins the Fermi level. The projected density of state is shown in Fig. S3. In accordance with PBE, SCAN functional gives similar result on the evolution of electronic structure with hole doping.

\section{Additional Result of DMFT}

In Fig. S4, we show the DFT+DMFT Fermi surfaces for pristine and doped compounds. The pristine compound consists of three Fermi surface sheets, namely the dominating $\alpha$ pocket due to $\mathrm{Ni}-3 d_{x^{2}-y^{2}}$ orbitals, the electron-type $\beta$ pocket around $\Gamma$, and the electrontype $\gamma$ pocket around $\mathrm{R}$. With 0.2 hole-doping, the $\beta$ pocket disappears, and the $\gamma$ pocket shrinks. Upon 0.4 hole doping, both $\beta$ and $\gamma$ pockets disappears.

In Fig. S5, we show the hybridization function in Matsubara frequency from DFT + DMFT calculations. In pristine compounds, both Ni-3 $d_{x^{2}-y^{2}}$ and Ni-3 $d_{z^{2}}$ orbitals show strong hybridization with the conduction electrons, as indicated by their divergent behavior with
Table S-I. Ni-3d orbital occupations and effective masses in both pristine and hole doped compounds for $V_{d c}=47.4$ $\mathrm{eV}$. The numbers within the brackets are the effective mass of respective orbitals calculated using $m^{*} / m=1 / Z=1-$ $\left.\frac{\partial \operatorname{Im}\left[\Sigma\left(i \omega_{n}\right)\right]}{\partial \omega_{n}}\right|_{\omega_{n} \rightarrow 0}$.

\begin{tabular}{c|c|c|c|c}
\hline \hline doping & $d_{x^{2}-y^{2}}$ & $d_{z^{2}}$ & $d_{z x / z y}$ & $d_{x y}$ \\
\hline 0.0 & $1.289(2.40)$ & $1.648(1.26)$ & $3.797(1.25)$ & $1.941(1.30)$ \\
0.1 & $1.267(2.58)$ & $1.651(1.28)$ & $3.826(1.27)$ & $1.944(1.32)$ \\
0.2 & $1.251(2.43)$ & $1.659(1.26)$ & $3.826(1.27)$ & $1.939(1.32)$ \\
0.3 & $1.243(2.43)$ & $1.666(1.29)$ & $3.824(1.28)$ & $1.932(1.33)$ \\
0.4 & $1.239(2.06)$ & $1.671(1.29)$ & $3.819(1.29)$ & $1.923(1.31)$ \\
\hline \hline
\end{tabular}

Table S-II. Ni-3d orbital occupations and effective masses in both pristine and hole doped compounds for $V_{d c}=51.84$ $\mathrm{eV}$. The numbers within the brackets are the effective mass of respective orbitals.

\begin{tabular}{c|c|c|c|c}
\hline \hline doping & $d_{x^{2}-y^{2}}$ & $d_{z^{2}}$ & $d_{z x / z y}$ & $d_{x y}$ \\
\hline 0.0 & $1.409(1.78)$ & $1.768(1.20)$ & $3.860(1.20)$ & $1.960(1.23)$ \\
0.1 & $1.379(1.85)$ & $1.780(1.20)$ & $3.884(1.20)$ & $1.963(1.23)$ \\
0.2 & $1.358(1.84)$ & $1.785(1.20)$ & $3.885(1.20)$ & $1.962(1.24)$ \\
0.3 & $1.346(1.79)$ & $1.788(1.20)$ & $3.883(1.20)$ & $1.959(1.23)$ \\
0.4 & $1.337(1.74)$ & $1.792(1.21)$ & $3.880(1.20)$ & $1.954(1.23)$ \\
\hline \hline
\end{tabular}

$\omega_{n} \rightarrow 0$. A small doping with 0.1 hole quickly suppresses the divergent behavior of both orbitals. The doping effect on $t_{2 g}$ orbital hybridization functions is much less prominent.

In Tab. S-I we show the orbital occupation and effective masses in both pristine and doped compounds, calculated with $V_{d c}=47.4 \mathrm{eV}$. Decreasing of $d_{x^{2}-y^{2}}$ effective mass from 0.1 hole/cell doping to 0.4 hole/cell doping can be observed, and a sudden drop from 0.3 hole/cell doping to 0.4 hole/cell doping can be identified. Similar decreasing of $d_{x^{2}-y^{2}}$ effective mass can also be identified in $V_{d c}=51.84 \mathrm{eV}$ calculations (Tab. S-II), however the sudden drop from 0.3 to 0.4 hole/cell doping is absent in $V_{d c}=51.84 \mathrm{eV}$ calculations.

In Tab. S-III, we show the statistic weight of sampled many-body configurations in the pristine and doped compounds for $V_{d c}=51.84 \mathrm{eV}$. Although the $d^{10} \underline{\mathrm{L}}$ configuration weight is substantially higher than $V_{d c}=47.4 \mathrm{eV}$ calculations, the non-monotonic doping effect can still be observed. Similarly, the nonmonotonic doping effect can also be identified from the Ni-3d occupation and crystal field splittings in $V_{d c}=51.84 \mathrm{eV}$ calculations (Fig. [S6).

Table S-III. Statistic weight of many-body configurations in pristine and doped compounds for $V_{d c}=51.84 \mathrm{eV}$.

\begin{tabular}{c|c|c|c|c|c}
\hline \hline doping & $d^{10} \underline{\mathrm{L}}$ & $d^{9}$ & $d_{x^{2}-y^{2}}^{9}$ & $d_{S=0}^{8}$ & $d_{S=1}^{8}$ \\
\hline 0.0 & $21.4 \%$ & $58.5 \%$ & $41.9 \%$ & $6.4 \%$ & $12.1 \%$ \\
0.1 & $21.2 \%$ & $59.5 \%$ & $45.3 \%$ & $6.8 \%$ & $11.1 \%$ \\
0.2 & $20.3 \%$ & $59.7 \%$ & $46.9 \%$ & $7.5 \%$ & $11.0 \%$ \\
0.3 & $19.8 \%$ & $59.7 \%$ & $47.8 \%$ & $8.1 \%$ & $10.9 \%$ \\
0.4 & $19.2 \%$ & $59.5 \%$ & $48.3 \%$ & $8.9 \%$ & $10.8 \%$ \\
\hline \hline
\end{tabular}


(a)

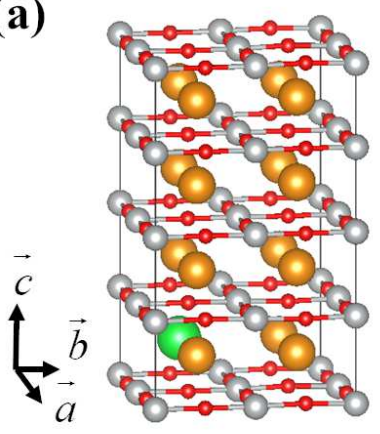

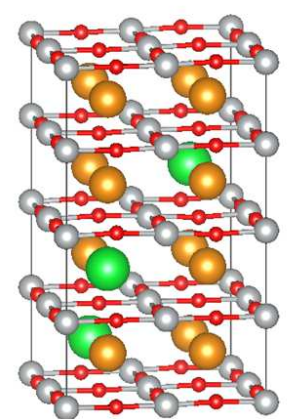

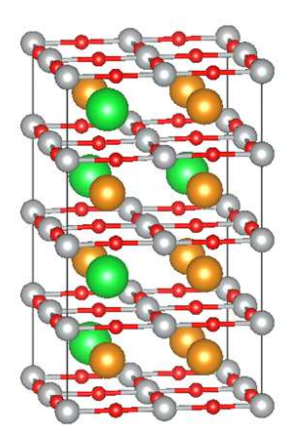

(b)

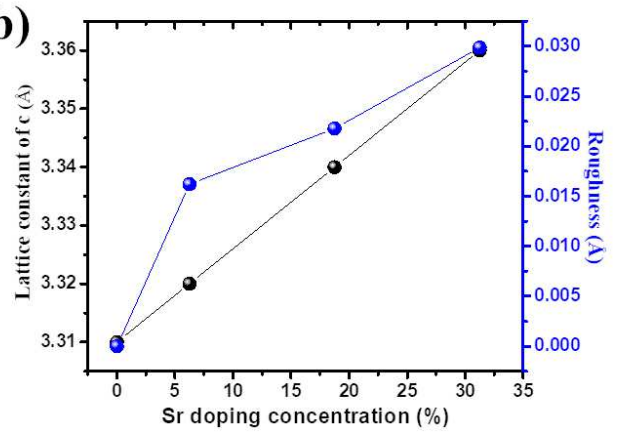

Figure S1. (a) From left to right: perspective view of $\mathrm{NdNiO}_{2}$ supercell with $\delta=6.25 \%, 18.75 \%$ and $31.25 \%$. The silver, red, yellow and green balls represent $\mathrm{Ni}, \mathrm{O}, \mathrm{Nd}$ and $\mathrm{Sr}$ atoms. (b) Lattice constant in c direction and roughness with respect to $\delta$.
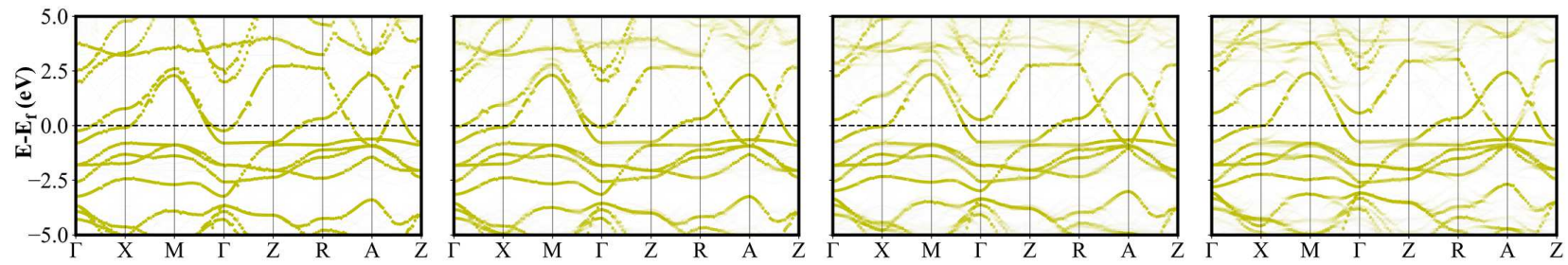

Figure S2. Unfolded band structures for different Sr doping concentration. From left to right: $\delta=0.00 \%, 6.25 \%, 18.75$ $\%$ and $31.25 \%$.
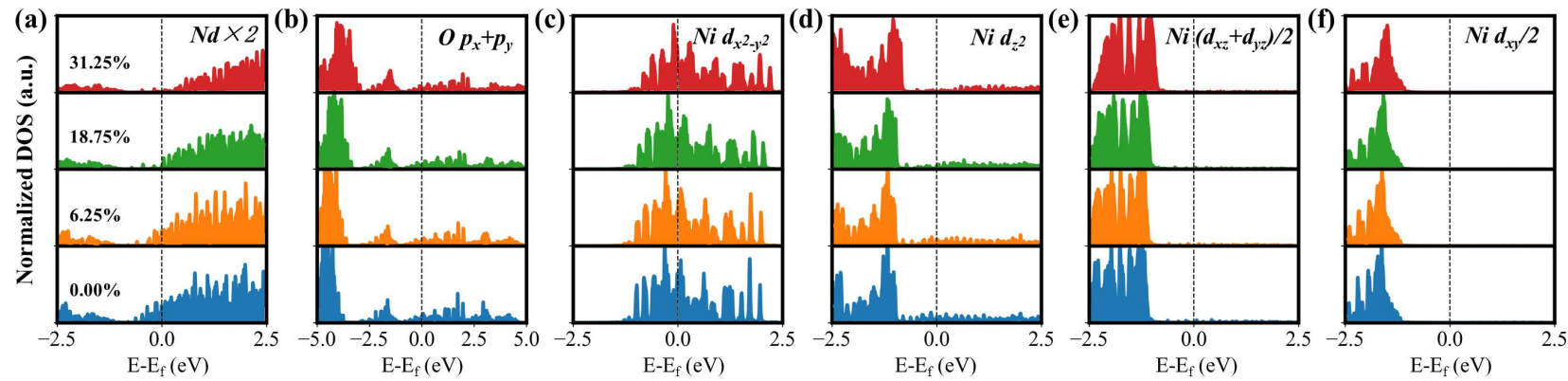

Figure S3. (a)-(f) Projected density of state with different $\delta$ on different subspaces, from left to right: Nd atoms, O $p_{x+y}$, $\mathrm{Ni} d_{x^{2}-y^{2}}$, Ni $d_{z^{2}}, \mathrm{Ni} d_{x z}+d_{y z}$ and Ni $d_{x y}$ orbitals. The weight in (a), (e) and (f) has been rescaled by a factor of $2,1 / 2$ and $1 / 2$. From bottom to top, $\delta$ increases from $0.00 \%$ to $31.25 \%$ in each panel.
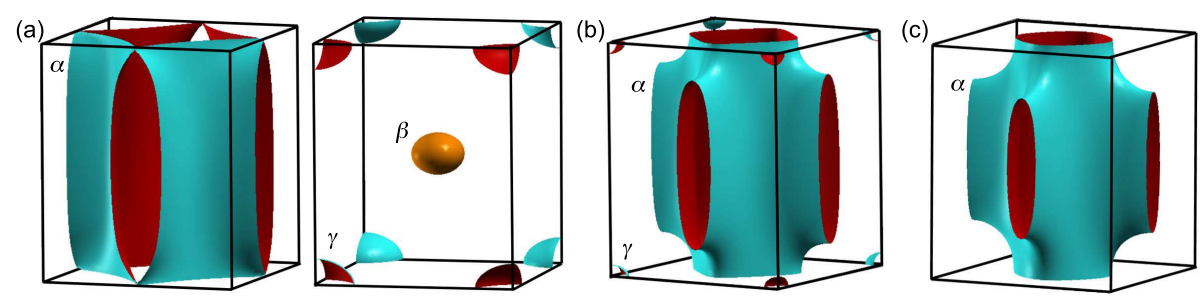

Figure S4. DFT + DMFT Fermi surfaces of (a) Pristine $\mathrm{NdNiO}_{2}$, (b) 0.2 hole-doped $\mathrm{NdNiO}_{2}$ and (c) 0.4 hole-doped $\mathrm{NdNiO}_{2}$ from $V_{d c}=47.4 \mathrm{eV}$ calculations. For clarity purposes, the dominating $\alpha$ sheet is separated from $\beta$ and $\gamma$ pockets in panel (a). 

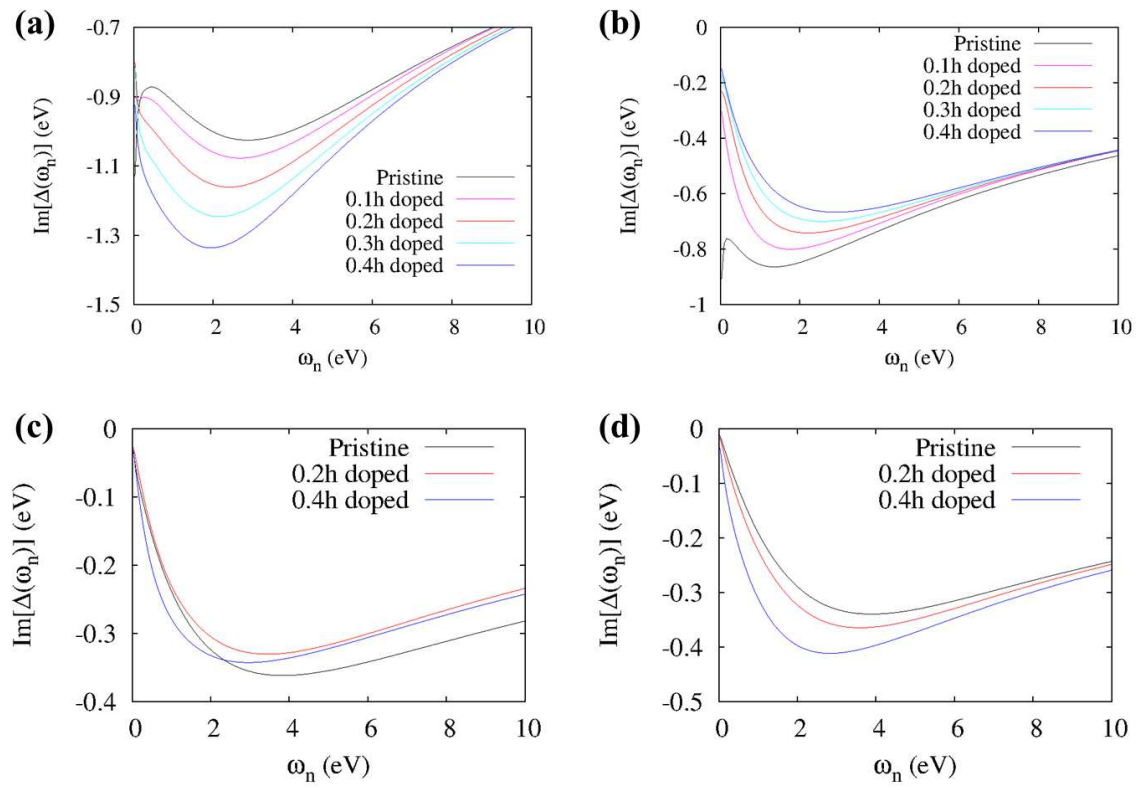

Figure S5. Doping dependence of hybridization function for (a) Ni-3 $3 d_{x^{2}-y^{2}}$, (b) Ni-3 $3 d_{z^{2}}$, (c) Ni-3 $d_{z x / z y}$, and (d) Ni-3dxy orbitals in $V_{d c}=47.4 \mathrm{eV}$ calculations.
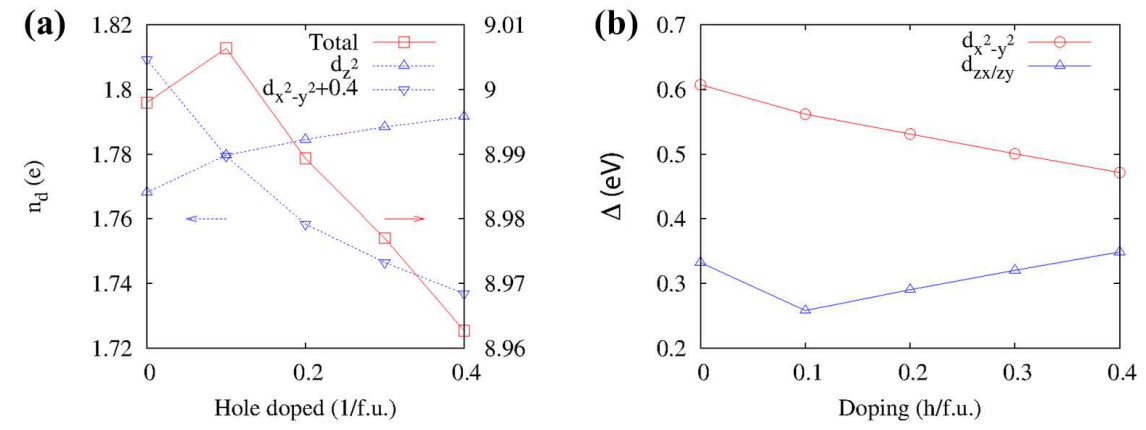

Figure S6. Evolution of (a) Ni-3d orbital occupation and (b) Ni-3d orbital crystal field splitting under hole doping, calculated using $V_{d c}=51.84 \mathrm{eV}$.

\section{Crystal field splitting of d orbitals}

Here we focus on the crystal field splitting of d orbitals in $\mathrm{NdNiO}_{2}$. To begin with, the splitting $O_{h}$ point group is shown in Fig. [S7(a), where $d_{x^{2}-y^{2}}, d_{z^{2}}$ are two-fold degenerated and $d_{x z}, d_{y z}, d_{x y}$ are three-fold degenerated. The $D_{4 h}$ point group can be obtained by moving the two vertical ligands to infinity as shown by the dashed line in Fig. S7(b). Since the d orbitals are anti-bonding type, such lifting of vertical ligands will weak the anti-bonding d orbitals, the result is orbital energy lowering as indicated by the green arrows in Fig. S7(b). The other two orbitals- $d_{x^{2}-y^{2}}, d_{x y^{-}}$ experience no affect for their planar orbital character. This picture can be modified by the apical anions as shown in Fig. S7(c), where $d_{z^{2}}, d_{x z}$ and $d_{y z}$ are pushed to higher energy. Therefore, the $d_{x y}$ now has the lowest energy while the $d_{x^{2}-y^{2}}$ has the highest energy. Such an orbital order will change with the inclusion of inter- action as shown in Fig. S7(d). The $d_{x^{2}-y^{2}}$ is the most correlated one and its orbital energy is even lower than $d_{z^{2}}$. Such a orbital order flip has also been reported in recent $\mathrm{GW}+\mathrm{DMFT}$ study [63].

\section{Electronic structure with f-electrons}

We show the results from calculation explicitly considering Nd-4 $f$ electrons in Fig. S8. These calculations are done with $U_{f}=6.0 \mathrm{eV}, J_{f}=0.7 \mathrm{eV}$ on Nd-4f orbitals and $U_{d}=5.0 \mathrm{eV}, J_{d}=0.8 \mathrm{eV}$ on Ni-3d orbitals. The k-resolved spectral functions are almost the same with the calculations without f-electrons (as shown in Fig. 3). In addition, the $3 d$-orbital occupation and effective masses are also qualitatively the same. Since the calculations are extremely expensive due to $\mathrm{Nd}$ $4 f$ orbitals, we have only calculated pristine and 0.2 hole-doped compounds. 

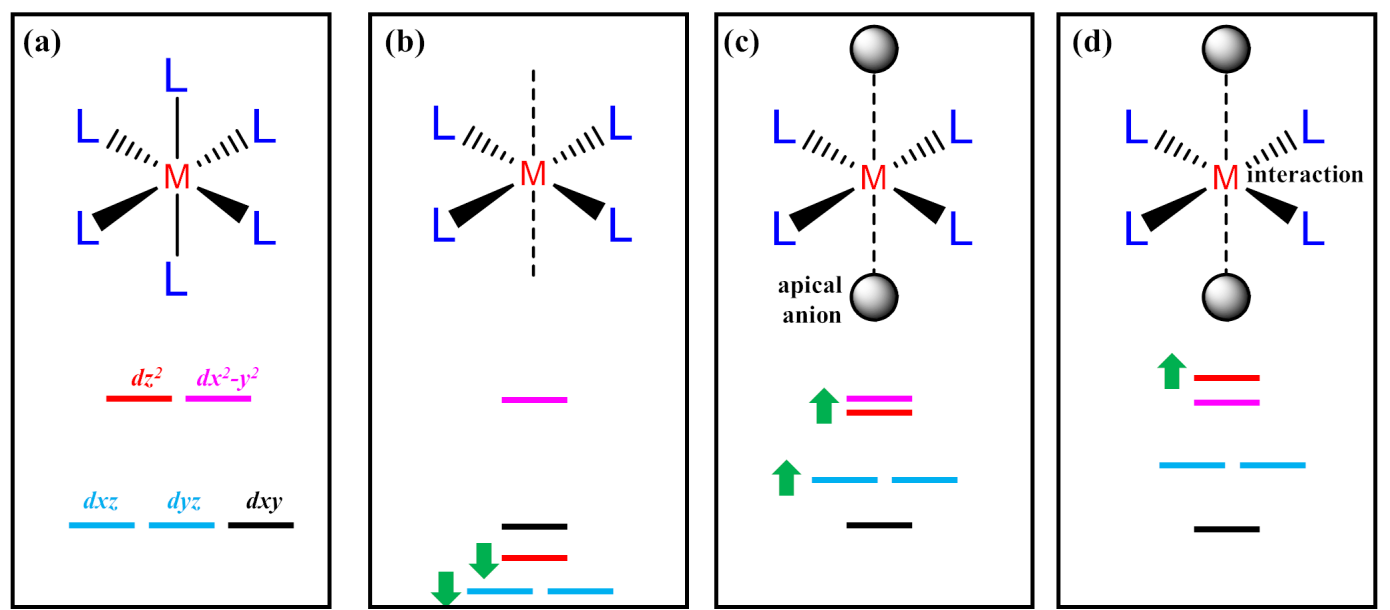

Figure S7. Crystal field splitting of d orbitals with (a) $O_{h}$ symmetry (b) $D_{4 h}$ (c) $D_{4 h}$ plus apical anions and (d) $D_{4 h}$ plus apical anions with interaction. The d orbitals are labelled by different colors: $d_{x^{2}-y^{2}}, d_{z^{2}}, d_{x z}\left(d_{y z}\right)$ and $d_{x y}$ orbital corresponds to pink, red, blue and black color.The greed filled arrows indicate how orbital order evolves with new effects.

Table S-IV. In a calculation with f-electrons, 3d-orbital occupations and effective masses in both pristine and 0.2-hole doped compounds. The numbers within the brackets are the effective mass of respective orbitals. .

\begin{tabular}{c|c|c|c|c}
\hline \hline doping & $d_{x^{2}-y^{2}}$ & $d_{z^{2}}$ & $d_{z x / z y}$ & $d_{x y}$ \\
\hline 0.0 & $1.271(2.30)$ & $1.631(1.23)$ & $3.775(1.21)$ & $1.942(1.23)$ \\
0.2 & $1.236(2.28)$ & $1.628(1.24)$ & $3.806(1.23)$ & $1.945(1.24)$ \\
\hline \hline
\end{tabular}

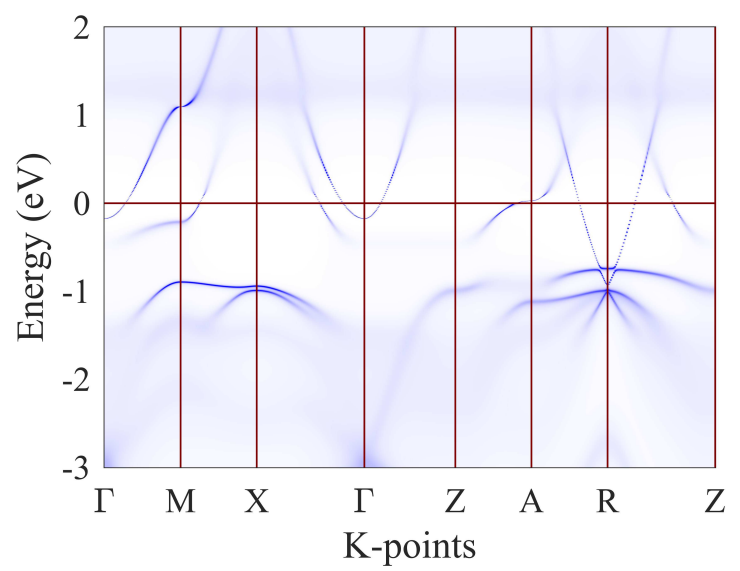

(a) Pristine

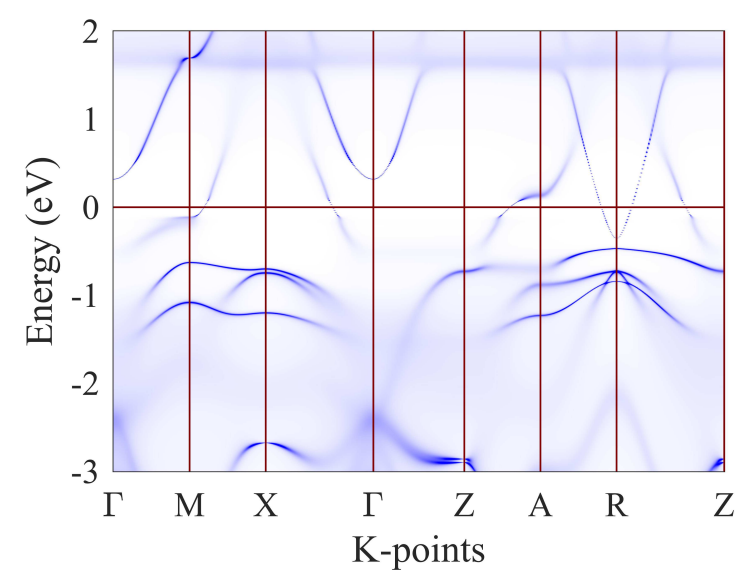

(b) Doped

Figure S8. k-resolved spectral function from DFT+DMFT calculations, by including f-elections. 